\title{
Sécrétion d'insuline : parcours intracellulaire et cheminement extracellulaire
}

Le cheminement de l'insuline a été suivi au travers des différents compartiments cellulaires, ainsi que son parcours extracellulaire vers les cellules cibles. Dans la cellule $B$ du pancréas, la pré-pro-insuline est synthétisée dans le réticulum endoplasmique rugueux et elle est ensuite transférée vers l'appareil de Golgi. Des granules "immatures" provenant des saccules golgiens concentrent la pro-insuline, la clivent en insuline et en Cpeptide et ils transportent ensuite ces peptides vers la membrane plasmique où s'effectue la décharge. Dans le milieu extracellulaire, l'insuline diffuse au travers de l'espace interstitiel et s'associe avec des sites localisés sur la membrane latérale de la cellule $B$ elle-même, sur les autres cellules pancréatiques endocrines et exocrines, ainsi que sur les cellules endothéliales des capillaires sanguins. Les cellules présentes sur le parcours extracellulaire de l'insuline sont exposées à des concentrations variables d'insuline à cause de la dilution rapide de l'hormone. Cette situation affecte particulièrement les cellules acineuses du pancréas dont la population se répartit en deux catégories selon leur position. Une nouvelle population de cellules $B$ localisées le long des épithéliums des canaux pancréatiques s'ajoute aux cellules B des îlots de Langerhans. Ces cellules sont de type ouvert et ont la capacité de sécréter l'insuline de façon " exocrine " dans le suc pancréatique. Il est donc possible que l'insuline véhiculée par le suc pancréatique exerce une action paracrine sur les cellules épithéliales thèse mérite d'être considérée pour mieux comprendre les problèmes du diabète. 


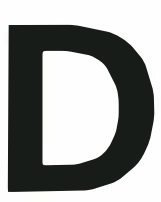

es travaux de recherches reposant sur l'application des techniques de fractionnement cellulaire et d'autoradiographie ont permis de clarifier les principaux mécanismes qui concourent à la synthèse et à la sécrétion de l'insuline [1]. Cette sécrétion, commune à toutes les cellules endocrines, est de nature mérocrine : elle comprend l'étape de synthèse d'une pré-prohormone, au niveau du réticulum endoplasmique rugueux ; après maturation en hormone active dans le système de golgien, elle est empaquetée dans des granules de sécrétion pour être déversée dans le milieu interstitiel. La technique d'autoradiographie introduit une dimension dynamique dans l'étude de la biologie cellulaire ; cependant, elle présente une grande faiblesse relativement à sa spécificité. En effet, tous les produits de synthèse cellulaire se trouvent marqués en même temps, ce qui entraîne un signal parfois complexe et difficile à interpréter. L'approche immunocytochimique présente par contre l'avantage d'une grande spécificité pour les protéines étudiées. En effet, les derniers progrès accomplis dans le domaine de l'immunocytochimie ultrastructurale permettent d'obtenir une grande spécificité dans la nature des molécules détectées, une excellente résolution dans l'identification des structures cellulaires marquées, ainsi qu'une quantification précise des intensités du marquage [2].

Dans le travail que nous présentons ici, nous avons profité de ces avantages pour réévaluer les étapes de sécrétion de l'insuline et de son cheminement extracellulaire vers les cellules cibles. Grâce à l'utilisation combinée d'anticorps spécifiques et du complexe protéine A-or colloïdal, nous avons pu mettre en évidence en microscopie électronique, la présence de molécules d'insuline et de Cpeptide au niveaux de différents compartiments et sous-compartiments cellulaires de la cellule B du pancréas [3], dans le tissu interstitiel environnant et dans la vascularisation sanguine du pancréas [4]. Nous avons également pu révéler l'interaction de l'insuline avec les différentes

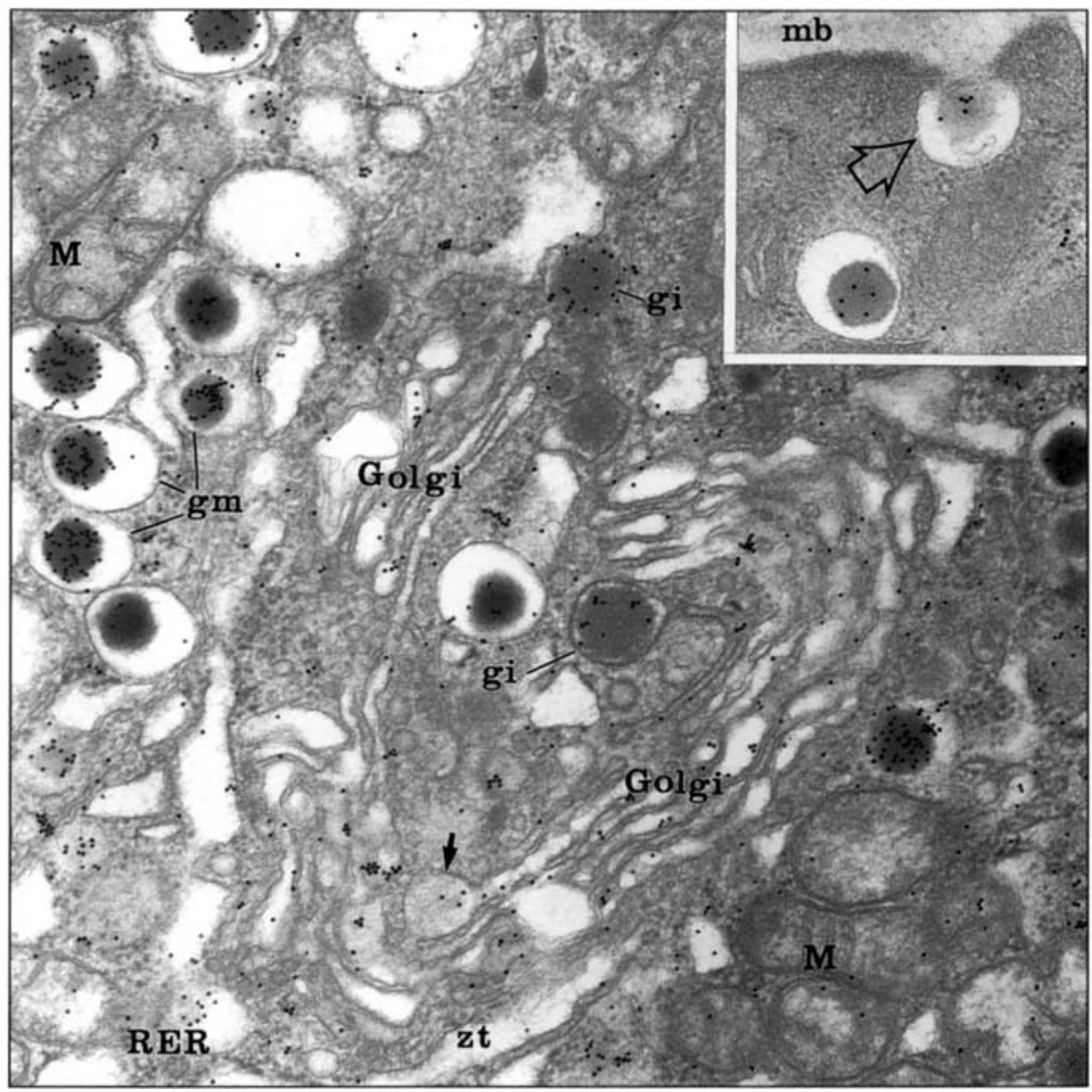

Figure 1. Région golgienne d'une cellule $B$ de pancréas de rat. L'insuline a été révélée par immunocytochimie à l'or colloïdal en employant un anticorps spécifique pour l'insuline et le complexe protéine A-or colloïdal Iréférences 2 et 3]. Le marquage par les particules d'or se retrouve en quantités différentes dans les saccules du réticulum endoplasmique rugueux (RER), dans ceux de la zone de transition (zt) et dans ceux de l'appareil de Golgi (Golgi). La flèche indique un granule "immature" en émergence à partir d'un des saccules de l'appareil de Golgi. Les granules de sécrétion "immatures " (gi) et " matures " (gm) sont marqués avec des intensités croissantes. L'encadré illustre un granule de sécrétion en état d'exocytose. $M$, mitochondries; mb, membrane basale. $\times 32000$. Encadré : $\times 36000$.

Au niveau tissulaire, l'insuline a été retrouvée uniquement dans les cellules B des îlots de Langerhans, ceux du pancréas ainsi que dans ceux de la paroi du duodénum [5].

Codée par son ARN messager, la pré-pro-insuline est une molécule de grande taille (11 500 daltons versus 9000 pour la pro-insuline et 6000 pour l'insuline). Elle apparaît dans la lumière du réticulum rugueux et se déleste rapidement de son peptide signal. L'ablation de ce peptide signal s'effectue sous l'action d'une endopeptidase présente dans les saccules du réticulum. La pro-insuline ainsi générée est transférée dans l'appareil de Golgi par le biais des éléments de la zone de transition; elle se déplace de la partie cis (zone de formation) vers la partie trans (zone de maturation) où sont élaborés les granules de sécrétion "immatures ". La proinsuline empaquetée dans ces granules quitte l'appareil de Golgi au niveau de sa zone de maturation (figure 1). Elle n'est scindée en ses deux composants, insuline et Cpeptide, qu'au niveau de ces granules immatures à membrane recouverte de clathrine, au cours donc d'une étape ultérieure au passage dans l'appareil de Golgi [6]. L'un des rôles attribués au C-peptide ou peptide de connexion, consiste à assurer le repliement adéquat ainsi qu'une 


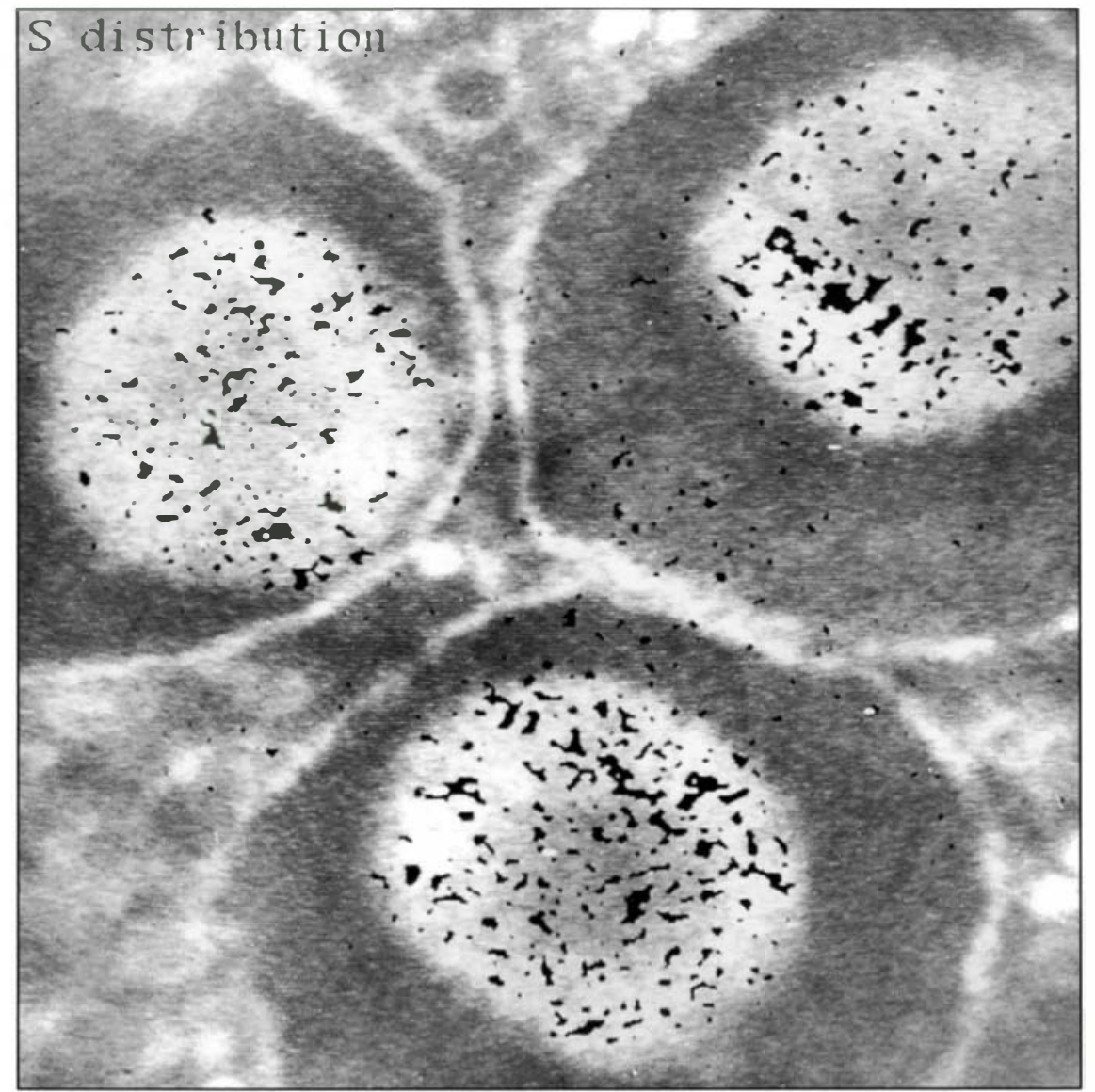

Figure 2. Démonstration par spectroscopie en perte d'énergie de la présence de soufre dans les granules de sécrétion d'insuline. Le signal obtenu en microscopie électronique analytique, par spectroscopie en perte d'énergie, se retrouve dans le cœur dense des granules où l'insuline est également localisée. $\times 218000$. l'espace périphérique délimité par la membrane du granule [3] (figure 3). La conversion de la pro-insuline n'est pas une condition indispensable à la sécrétion d'insuline. En effet, dans des conditions extrêmes, sous stimulation aiguë, cette conversion de proinsuline en insuline et C-peptide n'a pas le temps de se compléter; on aboutit alors à une sécrétion de la pro-hormone à activité physiologique inférieure. En dehors de ces compartiments cellulaires, l'insuline, de même que le $\mathrm{C}$-peptide, ont été retrouvés dans les corps crynophagiques (lysosomes), ce qui atteste de l'existence d'un système interne de dégradation, qui régularise la sécrétion en éliminant certains éléments non sécrétés par la cellule et permettant ainsi son renouvellement constant [3]. A la suite d'une stimulation appropriée, les granules se déplacent rapidement vers la surface cellulaire pour libérer leur contenu. Le système cytosquelettique, incluant microtubules et microfilaments, intervient dans cette translocation des granules. La décharge des deux composants, insuline et C-peptide, dans le milieu extérieur se fait de façon parallèle et prend place au niveau de la membrane plasmique par un mécanisme d'exocytose selon lequel on présume que la membrane du granule s'associe à celle de la cellule entraînant une fusion avec ouverture sur l'espace conformation tertiaire convenable à la molécule d'insuline par l'intermédiaire de ses ponts di-sulfures. La molécule d'insuline comporte en effet un grand nombre d'atomes de zinc et de soufre (figure 2). La maturation découle de l'action concomitante de l'hydrolyse menée par des enzymes dont l'action est semblable à celle de la trypsine et de la carboxypeptidase, et de l'acidification du contenu du granule [7] ; elle aboutit à la production d'insuline et de C-peptide en quantités équimolaires. La séparation physique de ces deux composants se poursuit dans les granules de sécrétion au cours de leur maturation et atteint un maximum quand l'insuline acquiert sa structure cristalline. A cette étape, l'insuline est concentrée dans le cour du granule alors que le C-peptide se trouve plutôt dans $\mathrm{m} / \mathrm{s} n^{\circ} 5$ vol. 9 , mai 93

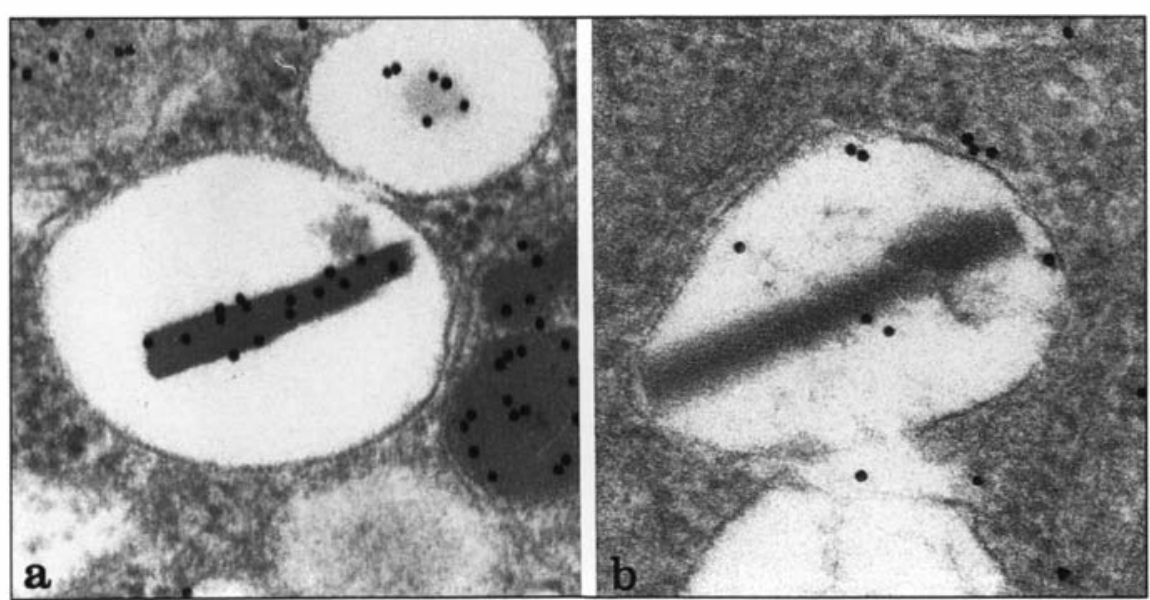

Figure 3. Localisation immunocytochimique à l'or colloïdal [références 2 et 3] de l'insuline (3a) et du C-peptide (3b) dans des granules de sécrétion matures. Ces granules présentent un cœur dense dans lequel l'insuline est cristallisée. Le marquage pour l'insuline est bien concentré au niveau du cœur cristallin (3a) tandis que celui pour le $C$-peptide se distribue dans l'espace entourant le cristal et délimité par la membrane du granule (3b). x 80000. 


\section{RÉFÉRENCES}

1. Orci L. The insulin factory : A tour of the plant surroundings and a visit to the assembly line. Diabetologia 1985; 28 : 528-46.

2. Bendayan M. Protein A-gold and protcin G-gold post-embedding immunoclectron microscopy. In : Hayat MA, eds. Colloidal Gold : Principles, methods and applications. San Diego : Academic Press, 1989 : 33-94

3. Bendayan M. Ultrastructural localization of insulin and C-peptide antigenic sites in rat pancreatic $B$ cell obtained by applying the quantitative high-resolution protein Agold approach. Am J Anat 1989; 185 205-16.

4. Bendayan M. Pathway of insulin in pancreatic tissue upon its release by the B-cell. Am J Physiol 1993 ; 264 : G187-G154.

5. Bendayan M, Park IS. Existence d'îlots de Langerhans au niveau de la paroi duodénale du rat. médecine/sciences 1991; 7 660-1.

6. Orci L,, Ravazzola M, Amherdt $M$, Madsen (), Vassali JI), Perrelet A. Direct identification of prohormone conversion site in insulin-secreting cells. Cell 1985; 42 : $671-81$

7. Orci L, Ravazzola M, Amherdt M, et al. Conversion of pro-insulin to insulin occurs coordinately with acidification of maturing secretory vesicles. J Cell Biol 1986 ; $103: 2273-81$.

8. Gold, G. Insulin structure and biosynthesis. In : Draznin B, Melmed S, LeRoith D), eds. Mol Cell Biol Diab I New York Alan R liss Inc, 1989 : 25-35.

9. Bonner-Weir S. Morphological evidence for pancreatic B-cell polarity within the isle of I angerhans. Diabetes 1988 ; 37 : 616-21.

10. Bendayan $M$. Association of secreted insulin with particular domains of the pancreatic B-cell plasma membrane : the actinrich microvilli. J Histochem Cytochem 1992 $40: 327-31$

11. Orci I,, Thorens B, Ravazzola M, Lodish $H F$. Localization of the pancreatic beta cell glucose transporter to specific plasma membrane domains. Science 1989 ; 245-95.

12. Argoud GM, Schade ISS, Eaton RP. Insulin suppresses its own secretion in vivo. Diabetes 1987 ; 36 : 959-62.

13. Draznin B, Goodman M, Leitner JW Sussman KE. Feedback inhibition of insulin on insulin secretion in isolated pancreatic islets. Endocrinology $1986 ; 118: 1054-8$.

14. Stagner J, Samois E, Polonsky K, Pugh $W$. Lack of direct inhibition of insulin secre tion by exogenous insulin in the canine pan-

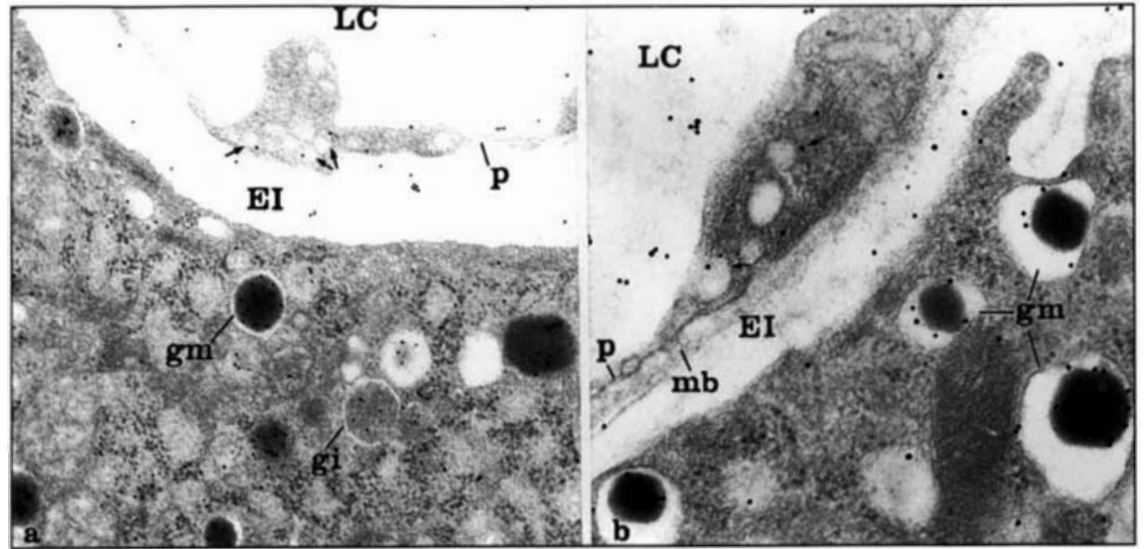

Figure 4. Localisation immunocytochimique à l'or colloïdal [références 2 et 3] de l'insuline (4a) et du C-peptide (4b) dans des cellules B du pancréas. Les figures illustrent les zones périphériques des cellules faisant face aux capillaires sanguins. Les marquages se retrouvent au niveau des granules de sécrétion " matures" (gm) et "immatures " (gi), dans l'espace interstitiel (EI) et dans la lumière des capillaires (LC). Les flèches indiquent la présence de marquage au niveau des vésicules plasmalemmales de l'endothélium capillaire. mb, membrane basale; p, pore. $\times 16000 . \times 32000$.

interstitiel. Des granules intimement accolés à la membrane plasmique ou même ouverts sur l'espace interstitiel sont parfois retrouvés, conférant une base morphologique à ce phénomène d'exocytose (figure 1). A partir de cette étape, on estime également que l'insuline aussi bien que le C-peptide diffusent dans l'espace interstitiel, traversent la paroi capillaire pour rejoindre, par la voie de la circulation sanguine, les différentes cellules cibles (figure 4). Deux phénomènes importants ponctuent le cheminement cellulaire de l'insuline de son site de synthèse à son site de décharge : la maturation, que l'on vient de décrire, et la concentration qui aboutit à la sécrétion de quantités considérables d'hormone. En effet, un fort gradient de concentration survient le long du cheminement intracellulaire, aboutissant à des concentrations de l'ordre de $10^{-2} \mathrm{M}$ d'insuline par granule de sécrétion [8]. Dans ce phénomène de concentration, l'immunocytochimie quantitative a contribué par des données tout à fait nouvelles et significatives [3]. En effet, la quantification du marquage immunocytochimique dans les différents compartiments et sous-compartiments cellulaires a révélé les sites où a lieu le phénomène de concentration de l'insuline [3]. Ceux-ci sont illustrés à la figure 5. Au niveau du réticulum, la pro-insuline converge et se concentre dans les saccules proches de la zone de transition, juste avant de passer dans l'appareil de Golgi. Par la suite, la pro-insuline est transférée de saccule en saccule sans concentration évidente jusqu'au cinquième et sixième saccule, dans la zone de maturation de l'appareil de Golgi où nous retrouvons une nouvelle étape de concentration. De ces derniers saccules, dérivent les granules de sécrétion. Tout d'abord "immatures" avec une couverture de clathrine, ils atteignent ensuite leur maturité tout en poursuivant le processus de concentration de l'insuline.

Peu d'études ont été consacrées au cheminement extracellulaire de l'insuline, particulièrement au travers du tissu conjonctif du pancréas et son transfert dans le sang au travers de l'endothélium capillaire. Nous avons récemment analysé et quantifié la distribution du marquage pour l'insuline dans l'espace interstitiel du pancréas [4]. Ce marquage apparaît assez homogène à travers le tissu interstitiel même si, parfois, une association préférentielle semble s'effectuer avec les fibres de collagène. Par contre on a constaté que les membranes basales - celles des cellules endocrines tout comme celles des capillaires sanguins - n'opposent aucune restriction au passage de l'insuline. Ces 


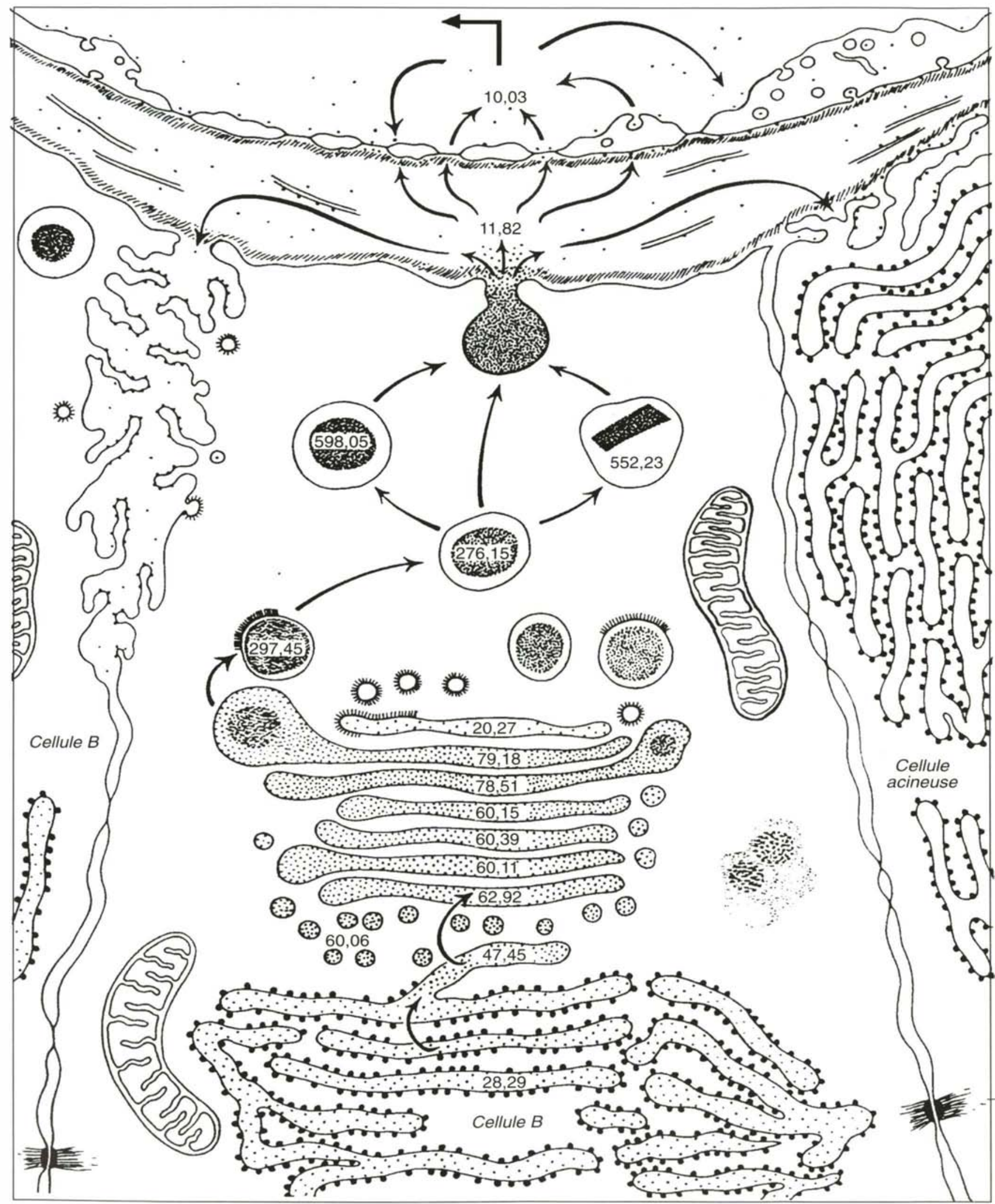

Figure 5. Schéma illustrant les différentes étapes de la synthèse et de la sécrétion de l'insuline par la cellule B du pancréas ainsi que le cheminement entrepris par l'insuline à sa sortie de la cellule. Les valeurs fournies correspondent aux intensités du marquage immunocytochimique pour l'insuline, tandis que les flèches indiquent le sens du parcours. Les petits points denses reflètent la présence d'insuline. 


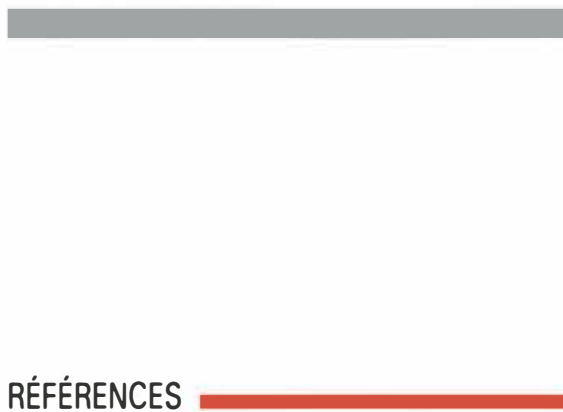

15. Sussman KE. Intraislet hormone regulation. In : Draznin B, Melmed S, LeRoith D eds. Mol Cell Biol Diab Mol I New York : Alan R Liss Inc, 1989: 117-23.

16. Verspohl EJ, Ammon HPT. Evidence for presence of insulin receptors in rat islets of Langerhans. J Clin Invest 1980 ; 65 : 1230-7.

17. Bergeron JJ, Rachubinski R, Searle N et al. Radioautographic visualization of in vivo insulin binding to the exocrine pancreas. Endocrinology $1980 ; 107$ : 1069-80.

18. Sakamoto C, Williams JA, Roach E Goldfine ID. In vivo localisation of insulin binding to cells of the rat pancreas. Proc Soc Exp Biol Med 1984; 175 : 497-502.

19. Bar RS, Hoak JC, Peacock ML. Insu lin receptors in human endothelial cells identification and characterization. I Clin Endocrinol Metab 1978 ; 47 : 699-702.

20. Frank HJL, Pardridge WM. A direc in vitro demonstration of insulin binding to isolated brain microvessels. Diabetes 1981 ; 30 : 757-61

21. King GL, Johnson SM. Receptormediated transport of insulin across endothelial cells. Science $1985 ; 227: 1583-6$.

22. Bendayan M. Anatomic basis of isletacinar interaction in the pancreas. Reg Pept Let $1990 ; 11: 1-7$

23. Bendayan M. Presence of endocrine cells in pancreactic ducts. Pancreas 1987 ; 2 : 393-7.

24. Park I-S, Bendayan M. Characterization of the endocrine cells in the pancreatic bile duct system of the rat. Anat Rec 1992 232 : 247-56.

25. Lawrence AM, Prinz RA, Paloyan E, Kokal WA. Glucagon and insulin in pancreatic exocrine secretions. Lancet 1979 ; 2 : 1354-5.

26. Colon JM, Rouiller D, Boden G Under RH. Characterization of immunocreactive components of insulin and somatostatin in canine pancreatic juice. FEBS Lett $1979 ; 105: 23-6$

27. Prinz RA, Kirstein L, Connick E, Paloyan E, Lawrence AM. Insulin and glucagon in human pancreatic exocrine fluid.

\begin{tabular}{|c|c|c|c|c|}
\hline \multicolumn{5}{|c|}{$\begin{array}{l}\text { INTENSITE* DU MARQUAGE IMMUNOCYTOCHIMIQUE } \\
\text { DE L'INSULINE DANS L'ESPACE INTERSTITIEL } \\
\text { ET LES CAPILLAIRES PANCREATIQUES }\end{array}$} \\
\hline & $\begin{array}{l}\text { Région } \\
\text { insulaire }\end{array}$ & $\begin{array}{c}\text { Région } \\
\text { pré-insulaire }\end{array}$ & $\begin{array}{c}\text { Région } \\
\text { télé-insulaire }\end{array}$ & Foie \\
\hline $\begin{array}{l}\text { Espace } \\
\text { interstitiel }\end{array}$ & $11,82 \pm 0,80$ & $6,74 \pm 0,46$ & $4,88 \pm 0,55$ & - \\
\hline $\begin{array}{l}\text { Lumière } \\
\text { capillaire }\end{array}$ & $10,03 \pm 0,42$ & $8,05 \pm 0,54$ & $5,84 \pm 0,48$ & $9 \pm 0,01$ \\
\hline
\end{tabular}

* nombre de particules d'or par $\mu \mathrm{m}^{2}$, valeurs moyennes \pm ESM.

résultats viennent étayer la présomption selon laquelle l'insuline une fois sécrétée par la cellule, diffuse assez librement dans l'interstice pour interagir avec les autres cellules pancréatiques ou pour rejoindre la circulation sanguine. Une association étroite de l'insuline avec les membranes plasmiques des cellules pancréatiques endocrines (y compris la cellule B ellemême) aussi bien qu'exocrines, ainsi qu'avec les membranes des cellules endothéliales des capillaires sanguins a été révélée [4]. En ce qui concerne les cellules à insuline, il a été établi qu'en terme de sécrétion, la cellule est polarisée $[9,10]$. La libération de l'insuline a lieu de préférence au niveau de la membrane cellulaire qui fait face aux capillaires sanguins. Cette membrane est relativement lisse et présente de nombreuses associations avec les granules de sécrétion [10]. Par contre, les membranes latérales des cellules B portent de nombreuses et courtes projections cytoplasmiques, reconnues comme des microvillosités riches en faisceaux de filaments d'actine [10]. La membrane de ces microvillosités détient des sites d'affinité et des récepteurs pour plusieurs composés, notamment l'insuline elle-même [10] et le transporteur du glucose [11]. Ces données ont été mis en évidence par immunocytochimie à haute résolution. La présence de récepteurs à insuline au niveau des membranes des cellules à insuline est certes discutée mais plusieurs phénomènes témoignent de leur existence qui est reliée à la fonc- tion de rétroaction qu'assure l'insuline dans la régulation de sa propre sécrétion [12-16]. La polarisation de la cellule $B$ et la spécialisation de sa membrane plasmique se résument donc en une partie apicale faisant face aux capillaires sanguins, assignée à la sécrétion et une partie basolatérale portant des récepteurs importants à sa fonction. En ce qui concerne les autres cellules pancréatiques, cellules endocrines non B et cellules acineuses, la présence et le rôle d'un récepteur à insuline ont été bien établis [16-18] ; nos données morphologiques viennent les confirmer et les illustrer. De même, les cellules endothéliales des capillaires semblent posséder des récepteurs à insuline [19-21] et, selon la distribution du marquage immunocytochimique obtenu [4], ces récepteurs seraient répartis tant sur les membranes luminales que sur les membranes abluminales. Les marquages pour l'insuline et le Cpeptide, ont été également retrouvés au niveau des pores endothéliaux, ainsi que dans les vésicules plasmalemmales [4] (figure 4). Ces résultats indiquent donc une association préférentielle de l'insuline à des sites d'affinité membranaire ou récepteurs spécifiques. Ils démontrent aussi que l'insuline, ainsi que le $\mathrm{C}$-peptide, traversent la paroi vasculaire par les pores endothéliaux ainsi que par le système vésiculo-tubulaire des cellules endothéliales. Les raisons qui justifieraient l'existence de deux voies de passage, l'une, celle des pores, probablement plus rapide que l'autre, 
celle par les vésicules plasmalemmales, restent cependant énigmatiques. La quantification des marquages obtenus dans les espaces extracellulaires nous a permis de préciser de nouvelles notions. En effet, dans l'espace extracellulaire le degré d'intensité du marquage de l'insuline est plus élevé près des cellules endocrines et s'estompe à mesure que l'on s'éloi-
Cette série d'observations reflète la dilution rapide de l'hormone survenant à partir du site de sécrétion jusqu'au site d'action. Ainsi, suivant leur emplacement topographique, les tissus qui jalonnent le parcours de l'insuline, se trouvent exposés à des concentrations variables de l'hormone. Les cellules acineuses du pancréas en particulier, sont exposées à

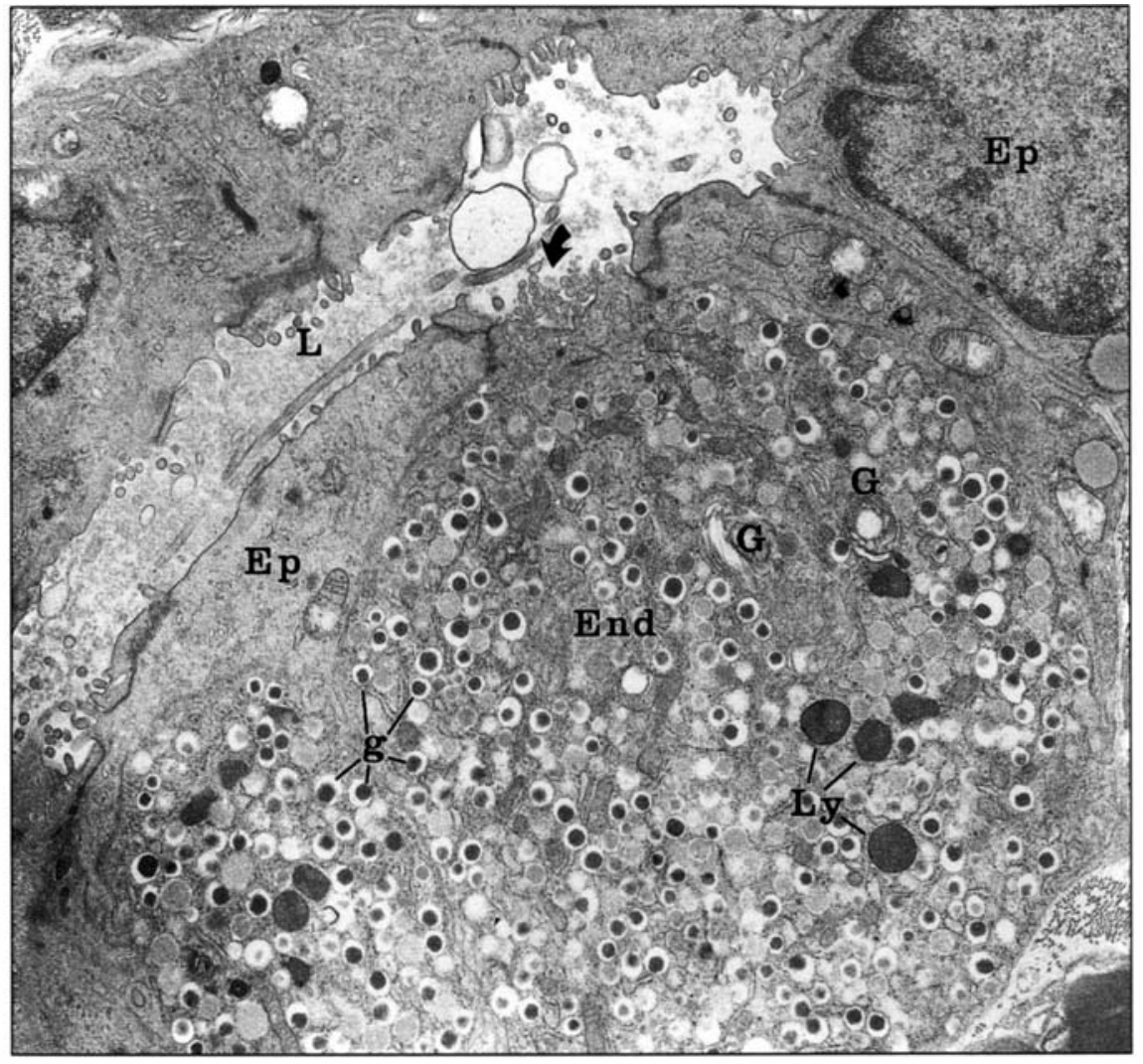

Figure 6. Paroi d'un canal pancréatique possédant une cellule endocrine à insuline (End) intercalée entre deux cellules épithéliales (Ep). La région apicale de cette cellule à insuline (flèche est en contact avec la lumière du canal (L). La cellule présente une morphologie classique avec un grand nombre de granules de sécrétion (g), des appareils de Golgi (G) et des lysosomes $(L y) . \times 5000$.

gne des îlots de Langerhans [4]. De même, l'intensité des marquages obtenus dans la lumière des vaisseaux sanguins, élevée au niveau des capillaires insulaires, diminue progressivement dans les capillaires du tissu acinaire, à mesure que l'on s'éloigne des îlots et que l'on sort du pancréas ; elle atteint des valeurs très basses dans les vaisseaux du foie (Tableau I). n leur éloignement de Deux zones différentes ont été identiflees dans le parenchyme acinaire selon leur position par rapport aux îlots de Langerhans : la zone périinsulaire, qui comprend les acini entourant les îlots, et la zone téléinsulaire qui inclut les acini situés loin du tissu endocrine [22]. Les cel- lules acineuses de ces deux zones comportent de notables différences : leur taille, leurs contenus enzymatiques et leurs réponses aux stimuli [22]. Ces différences sont attribuables aux effets de leurs exposition à des concentrations différentes d'insuline.

Si les cellules B sécrétant l'insuline font classiquement parties des îlots de Langerhans, nous venons, par ailleurs, de décrire et de caractériser des cellules isolées, mais nombreuses, dans la paroi des canaux pancréatiques [23, 24]. Ces cellules endocrines sont en tous points semblables à celles des îlots, sauf en ce qui concerne leur topographie. Elles sont localisées dans l'épithélium même, intercalées entre les cellules épithéliales (figure 6). Elles se retrouvent en nombres variables, tout le long du système des canaux excréteurs, depuis les canaux intercalaires jusqu'au canal de Wirsung qui débouche dans la lumière duodénale. Les cellules à insuline de ce système de canaux, tout comme les cellules à somatostatine d'ailleurs, sont de type ouvert avec une membrane plasmique "luminale " en contact avec la lumière du canal. Ces cellules peuvent avoir une sécrétion " exocrine" dans le suc pancréatique. En effet, des essais biochimiques radio-immunologiques ont démontré la présence de quantités importantes d'insuline dans le suc pancréatique [25, 26, 27]. Le rôle de l'insuline ainsi véhiculée vers la lumière intestinale, n'est pas bien établi. Cependant des travaux ont rapporté que l'insuline et la somatostatine pouvaient exercer des rôles paracrines sur le tissu épithélial des canaux pancréatiques et de l'intestin; les résultats de ces recherches sont importants car ils assignent des fonctions possibles à cette sécrétion " exocrine".

La compréhension des processus de sécrétion de l'insuline, des mécanismes de transport intra et extracellulaire ainsi que des interactions avec les cellules cibles est primordiale. Plusieurs étapes le long du cheminement de l'insuline, peuvent subir des altérations entraînant les déficiences hormonales propres aux diabètes. Des anomalies dans le déclenchement de la synthèse d'insuline se retrouvent lors d'une baisse de la sensibilité des glucorécepteurs. Des altérations du 
système adénylate cyclase entraîneraient des perturbations dans les métabolismes du calcium et par conséquent dans la fonction du cytosquelette. Des altérations de la maturation de la pro-insuline et des perturbations du processus de sécrétion provoquent des accumulations aberrantes de granules "immatures" qu'on retrouve dans le modèle diabétique du rat des sables Psammomys obesus (Ziv et Bendayan, résultats non publiés). Les altérations du système microtubulaire entraînant des déficiences dans la sécrétion se retrouvent dans le modèle de la souris Acomys cahirinus [28]. La paroi des vaisseaux sanguins subit des modifications majeures lors de la microangiopathie diabétique, ce qui pourrait perturber le transfert des hormones du milieu interstitiel vers le sang et vice versa [29]. Nous avons récemment démontré que l'insuline présente dans la lumière intestinale peut être absorbée par les cellules épithéliales entérocytaires et transportée, sans dégradation, par transcytose au côté baso-latéral [30]. Une fois déversée dans le milieu interstitiel de la muqueuse intestinale, l'insuline gagne rapidement la circulation sanguine. Cette boucle entéro-pancréatique fait actuellement l'objet de recherche pour être utilisée comme alternative thérapeutique dans l'administration d'insuline chez les patients diabétiques [30]. D'où l'importance d'une bonne analyse des étapes intervenant dans la sécrétion et dans le parcours des hormones, du site de synthèse au site d'action, pour parvenir à une meilleure compréhension des phénomènes physiopathologiques et menant au développement de nouveaux traitements

\section{Remerciements}

Ce travail a bénéficié du soutien financier du Conseil de Recherches Médicales du Canada.

\section{TIRÉS A PART}

M. Bendayan.

\section{RÉFÉRENCES}

28. Malaisse-Lagae F, Ravazzola M, Amherdt M, Gutzeit A, Stauffacher W, Malaisse WJ, Orci L. An apparent abnormality of the B-cell microtubular system in spiny mice (Acomys cahirinus). Diabetologia 1975 ; $11: 71-6$.

29. Williamson JR, Kilo C. Basement membrane in diabetes mellitus. In : Draznin B, Melmed S, LeRoith D eds. Mol Cell Biol Diab Mol III New York : Alan R Liss Inc, $1989:$ 19-29.

30. Bendayan M, Ziv E, Ben-Sasson R, Bar-On H, Kidron M. Morphocytochemical and biochemical evidence for insulin absorption by the rat ileal epithelium. Diabetologia $1990 ; 33$ : 197-204.

\section{Summary}

Insulin secretion : intracellular pathway and extracellular route

Insulin and C-peptide have been revealed by immunocytochemistry in the pancreatic tissue.

Combination of good ultrastructural preservation leading to immunolabelings of high resolution with quantitative evaluations, has permitted to follow the journey of insulin along the cellular compartments of the B-cell involved in secretion as well as along its extracellular path, revealing interactions of the secreted insulin with various pancreatic cells. In B-cells the prepro-insulin is synthesized in the rough endoplasmic reticulum, and the pro-insulin is transferred to the Golgi apparatus. Immature secretory granules originating from the Golgi saccules concentrate the proinsulin, convert it to insulin and C-peptide and along with maturation move them towards the plasma membrane for discharge. Outside the cell, insulin diffuses through the interstitial space and interacts with binding sites located in the lateral membrane of the Bcell itself and with other cells including non-B endocrine cells, acinar cells and the endothelial cells. Insulin appears to traverse the capillary wall through two rou- tes, the endothelial pores and the trans-endothelial vesicular system. A quantitative evaluation has revealed the rapid dilution of insulin as it travels through the interstitial space and the blood vessels. Cells present along the extracellular pathway are thus exposed to variable levels of insulin which influence differently their behavior. This is particularly true for the acinar cells which have been divided into two subpopulations according to their topographical location. In addition to their presence in the islets of Langerhans, B-cells were also revealed in the epithelial lining of the pancreatic duct system. These cells are of the " open " type and have the capability of an " exocrine " secretion of insulin into the pancreatic juice. A paracrine influence of the insulin conveyed by the pancreatic juice, upon the duct and intestinal epithelia has been suggested. Thus, the secretory pathway as well as the extracellular route of insulin, as revealed by high resolution immunocytochemistry, demands reevaluation for a better understanding of problems occurring in diabetes. 